\title{
Delayed Nontuberculous Mycobacterium Manifestation 1 Year after a Dog Bite on the Hand
}

\author{
Jong Keun Song (1), Jeong Hwan Shin (10), Jun Yong Lee (1) \\ Department of Plastic and Reconstructive Surgery, College of Medicine, The Catholic University of Korea, Seoul, Korea
}

\begin{abstract}
Nontuberculous mycobacteria infection can show various clinical manifestations as over 190 different species of nontuberculous mycobacteria have been identified. Among them, skin and soft tissue infections are commonly related with invasive procedures or trauma. The typical incubation period of rapidly growing mycobacteria is 3 to 6 weeks, whereas slowly growing mycobacteria takes 32 to 265 days of incubation. There have been a few cases of nontuberculous mycobacterium skin and soft tissue infections associated with animal bites, but none of them, not even mycobacteria with slow growth, had incubated for more than a year from the initial trauma. We present our case of delayed manifestation of nontuberculous mycobacterium skin and soft tissue infections at a year after receiving a dog bite on the hand. This case serves as a reminder that patients with delayed onset of local inflammation and granulation tissue on the hand must be reviewed for history of any previous trauma at the wound site and evaluated for the possibility of concealed nontuberculous mycobacterium infection.
\end{abstract}

Keywords: Nontuberculous mycobacterium; Long incubation period; Hand injury; Bites and stings

\section{Introduction}

The nontuberculous mycobacteria (NTM) is defined as any mycobacterial pathogen excluding Mycobacterium tuberculosis or Mycobacterium leprae. Because many NTM species have been identified, diagnosis is difficult and successful treatment for NTM infection is also complicated [1]. Though aerosol inhalation and water distribution systems are known as the main transmission routes of NTM disease, skin and soft tissue NTM infection is commonly related with invasive procedures or trauma [2-4]. Their incubation periods can be distinguished by their growth rates on agar [2]. Accordingly, NTM can be classified into slow-growing mycobacteria (SGM) with usual incubation periods of 32 to 265 days and rapidly growing mycobacteria (RGM) with typical incubation periods of 3 to 6 weeks [5]. Among the RGM species, Mycobacterium fortuitum and Mycobacterium chelonae are more commonly associated with skin and soft tissue infections (SSTIs), which are associated with animal exposure [3].

There are a few cases of nontuberculous mycobacterium SSTIs associated with animal bites, but to the best of our knowledge, none of them had an incubation period of more than a year from the initial trauma, not even SGM infection. Therefore, we present the first case of delayed manifestation of NTM infection after a year of incubation. The patient provided informed consent for inclusion in the study, and this study was approved by The Institutional Review Board of the Catholic University of Korea (IRB No. OC19ZESI0048).

\section{Case Report}

Received: November 2, 2019

Revised: November 6, 2019

Accepted: November 6, 2019

Corresponding author: Jun Yong Lee, M.D., Ph.D.

Department of Plastic and Reconstructive Surgery, Incheon St. Mary's Hospital, College of Medicine, The Catholic University of Korea, 56 Dongsu-ro, Bupyeong-gu, Incheon 21431, Korea

Tel: +82-32-280-5184

Fax: +82-32-510-2370

E-mail: ps.jylee@catholic.ac.kr

This is an Open Access article distributed under the terms of the Creative Commons Attribution Non-Commercia License (https://creativecommons.org/licenses/by-nc/4.0/) which permits unrestricted non-commercial use, distribution, and reproduction in any medium, provided the original work is properly cited.

(c) 2020 Korean Wound Management Society 


\section{Case}

A 37-year-old woman with no underlying disease had noted two adjacent masses on the third web space of her hand for a month (Fig. 1). The lesions showed infectious granulation tissue with a small opening producing serosanguinous discharge. The masses had repeatedly grown and reduced in size over the past month. The patient had not received any specific treatment at her local clinic but had dressed the wound with bandages herself. She also complained of mild pain when she used her hand. The patient recalled that she sustained a dog bite injury a year earlier, and the wounds were healed within a week without dressing. The abovementioned masses coincided with the points of penetration by the dog's teeth. Based on the trauma history of the patient and observation of infective granulation tissue, this was believed to be an NTM infection, and therefore polymerase chain reaction (PCR) and a staining test were added to excisional biopsy (Fig. 2). Operative findings
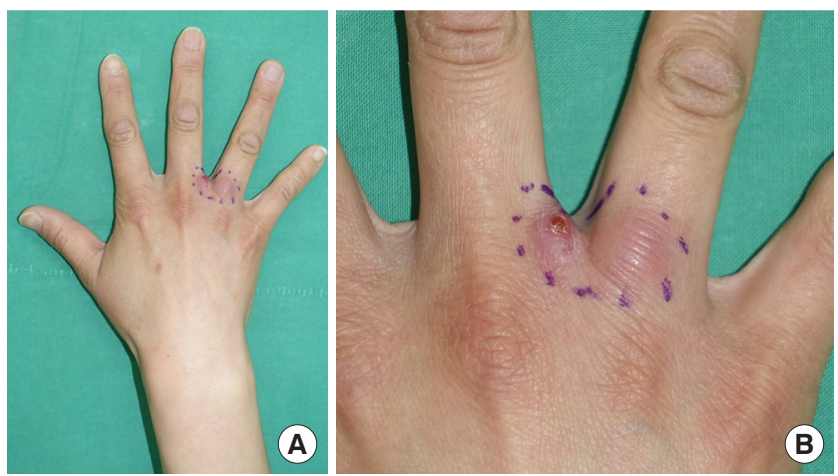

Fig. 1. Photographs taken at the initial presentation. (A, B) The skin layer was very fragile and thin.

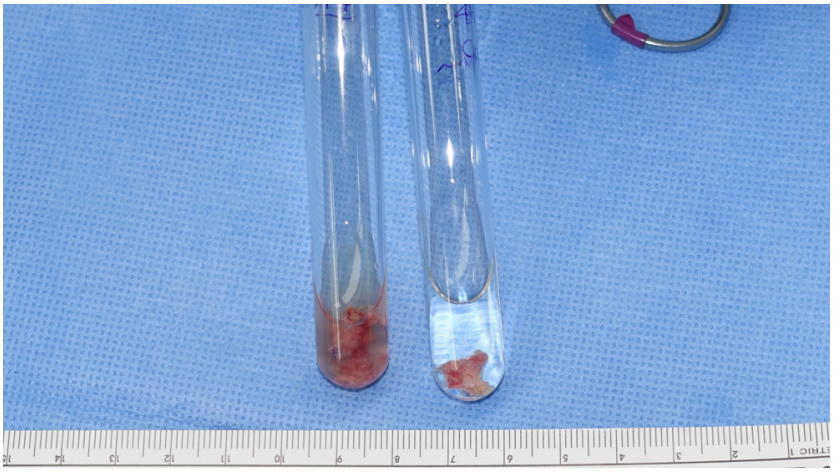

Fig. 2. Specimen from initial operation. The specimen in the right bottle was from the radial side of the wound and the left bottle was from the ulnar side of the wound. Biopsy and polymerase chain reaction test were performed on these specimens. showed a very fragile and thin layer of skin covering the granulation tissue, with peripheral induration. The granulation tissue cavities were filled with jelly-like discharge. Because the masses and amounts of discharge were small, only a small amount of specimen could be harvested. To avoid false negative findings due to the suppression of NTM, cefdinir (100 mg; three times a day) was administered after excisional biopsy. Initial Gram staining and acid-fast bacillus (AFB) staining presented negative findings. The results of NTM culture and drug susceptibility test failed to isolate species or susceptibility. The biopsy results were concluded as an abscess, whereas the PCR test showed NTM positive (cycling threshold, 33.10) and M. tuberculosis negative results.

Based on the PCR results, the antibiotics were replaced from cefdinir (100 mg; three times a day) to moxifloxacin (400 mg; once a day) and clarithromycin (500 $\mathrm{mg}$; twice a day) as prescribed by an infection specialist. Because only a small amount of the strain was available, genotyping of the NTM failed. Subsequent NTM sequencing tests showed insignificant results due to mixed peaks.

The patient was given sterile foam dressing every other day, but yellowish discharge was consistently noted. Hence, wound healing was delayed with continuous recurrent granuloma formation. Two additional debridements were performed 20 days and 2 months after the initial operation. Though the wound had healed completely on the 66th day, antibiotics were used for 6 months after the initial surgery. The patient was followed up every month. No further signs of infection or granuloma formation were observed and the patient reported no pain when she used her hand 6 months (Fig. 3).
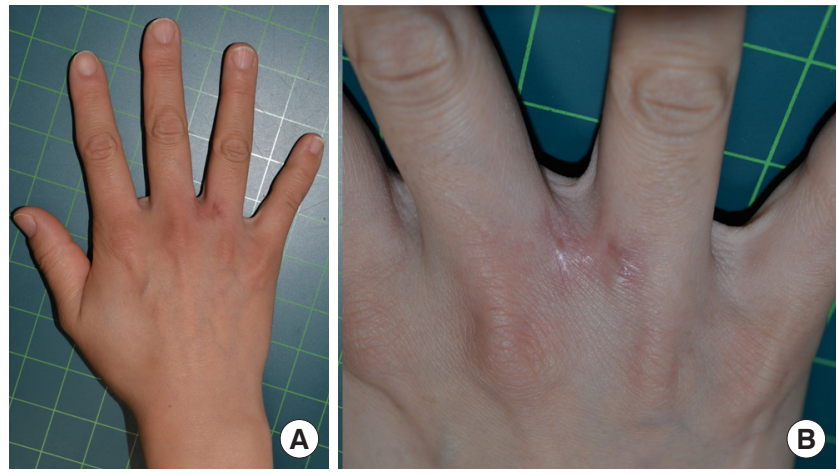

Fig. 3. Photographs taken at 6 months after treatment. (A, B) No further signs of infection or granuloma formation were observed and the patient reported no pain when using her hand. 


\section{Discussion}

The increasing number of reports of nontuberculous mycobacterium SSTIs in recent years has attracted significant attention in the medical community [6]. The modes of NTM transmission include (1) aerosolization and inhalation, (2) swallowing and aspiration, and (3) introduction into wounds, either via injury or surgery (or both) [7]. Only a few cases of infection with NTM species in humans due to animal bites have been reported in the available literature. These include infections transmitted by unusual penetration injuries such as bee stings or bird bites [2]. Although mycobacteria colonize in the oral flora, the main cause of NTM SSTIs is invasion of environmental mycobacteria through penetrating injuries from animal bites, not from oral flora [2]. Nevertheless, there are no reports of delayed NTM infections occurring after a year from the initial animal bite. Hence, we report the first case of NTM infection occurring in a healthy patient after an incubation period of a year. The immune status of the affected host factors significantly in the development of diseases caused by NTM; mycobacterium is suppressed for a long time in patients with healthy immune systems [1]. Therefore, it can be assumed that it may be expressed after a long time from initial trauma. This was supposedly the mechanism in our patient.

SSTIs associated with NTM can be challenging to diagnose due to a wide clinical spectrum of presentation, which includes both localized and disseminated forms of the disease $[5,8]$. For patients who have trauma history and chronic skin infection with granulation tissue, diagnostic workup related to NTM infection such as AFB smear and culture, biopsy and PCR should be added. PCR with nucleic acid amplification can detect mycobacteria deoxyribonucleic acid even in smearnegative specimens and paucibacillary tissue specimens that fail to grow on culture [5].

Because some nontuberculous mycobacterium SSTIs can take months to develop, the initial trauma may not be recalled [5]. Therefore, surgeons should ask in detail about the patient's past history. In this study, NTM genotyping and NTM sequencing test bore no meaningful results, making it difficult to determine the appropriate medication. We assume that it was difficult to obtain sufficient specimens during surgery because NTM-infected lesions had been suppressed for a long time. Finding out how to identify NTM genotyping with a small sample is another challenge.

This case shows that skin-penetrating animal bite injuries may cause delayed NTM infection after a year from the initial injury. It serves as a reminder for hand surgeons to suspect NTM infection and check for old trauma history, specifically if the patients have history of animal bites and chronic skin infection with granulation tissue on the hand. If NTM infection is suspected based on trauma history and clinical features, medical staff should perform a biopsy and PCR test to confirm NTM infection and perform appropriate surgical resection and administer antibiotics after obtaining the specimen.

\section{Conflict of interest}

No potential conflicts of interest relevant to this article are reported.

\section{ORCID iDs}

Jong Keun Song https://orcid.org/0000-0002-7117-9445

Jeong Hwan Shin https://orcid.org/0000-0001-9752-8941

Jun Yong Lee https://orcid.org/0000-0002-6967-4447

\section{References}

1. Baldwin SL, Larsen SE, Ordway D, et al. The complexities and challenges of preventing and treating nontuberculous mycobacterial diseases. PLoS Negl Trop Dis 2019;13: e0007083.

2. Iyengar KP, Nadkarni JB, Gupta R, et al. Mycobacterium chelonae hand infection following ferret bite. Infection 2013; 41:237-41.

3. Larson JM, Gerlach SY, Blair JE, et al. Mycobacterium chelonae/abscessus infection caused by a bird bite. Infect Dis Clin Pract 2008;16:60-1.

4. Wagner D, Young LS. Nontuberculous mycobacterial infections: a clinical review. Infection 2004;32:257-70.

5. Wang SH, Pancholi P. Mycobacterial skin and soft tissue infection. Curr Infect Dis Rep 2014;16:438.

6. Gonzalez-Santiago TM, Drage LA. Nontuberculous mycobacteria: skin and soft tissue infections. Dermatol Clin 2015; 33:563-77.

7. Falkinham JO 3rd. Environmental sources of nontuberculous mycobacteria. Clin Chest Med 2015;36:35-41.

8. Griffith DE, Aksamit T, Brown-Elliott BA, et al. An official ATS/IDSA statement: diagnosis, treatment, and prevention of nontuberculous mycobacterial diseases. Am J Respir Crit Care Med 2007;175:367-416. 